\title{
Acinetobacter baumannii invades epithelial cells and outer membrane protein A mediates interactions with epithelial cells Chul Hee Choi ${ }^{1}$, Jun Sik Lee ${ }^{1}$, Yoo Chul Lee ${ }^{1}$, Tae In Park² and Je Chul Lee*1
}

\author{
Address: ${ }^{1}$ Department of Microbiology, Kyungpook National University School of Medicine, Daegu, 700-422, Korea and ${ }^{2}$ Department of \\ Pathology, Kyungpook National University School of Medicine, Daegu, 700-422, Korea \\ Email: Chul Hee Choi - chchoi@knu.ac.kr; Jun Sik Lee - immunelee@knu.ac.kr; Yoo Chul Lee - yclee@knu.ac.kr; Tae In Park - tipark@knu.ac.kr; \\ Je Chul Lee* - leejc@knu.ac.kr \\ * Corresponding author
}

Published: 10 December 2008

BMC Microbiology 2008, 8:216 doi:10.1186/147|-2180-8-216
Received: 2 September 2008

Accepted: 10 December 2008

This article is available from: http://www.biomedcentral.com/I47I-2180/8/216

(C) 2008 Choi et al; licensee BioMed Central Ltd.

This is an Open Access article distributed under the terms of the Creative Commons Attribution License (http://creativecommons.org/licenses/by/2.0), which permits unrestricted use, distribution, and reproduction in any medium, provided the original work is properly cited.

\begin{abstract}
Background: Acinetobacter baumannii is a nosocomial pathogen of increasing importance, but the pathogenic mechanism of this microorganism has not been fully explored. This study investigated the potential of $A$. baumannii to invade epithelial cells and determined the role of $A$. baumannii outer membrane protein $A(A b O m p A)$ in interactions with epithelial cells.

Results: A. baumannii invaded epithelial cells by a zipper-like mechanism, which is associated with microfilament- and microtubule-dependent uptake mechanisms. Internalized bacteria were located in the membrane-bound vacuoles. Pretreatment of recombinant AbOmpA significantly inhibited the adherence to and invasion of $A$. baumannii in epithelial cells. Cell invasion of isogenic AbOmpAmutant significantly decreased as compared with wild-type bacteria. In a murine pneumonia model, wild-type bacteria exhibited a severe lung pathology and induced a high bacterial burden in blood, whereas AbOmpA- mutant was rarely detected in blood.

Conclusion: A. baumannii adheres to and invades epithelial cells. AbOmpA plays a major role in the interactions with epithelial cells. These findings contribute to the understanding of $A$. baumannii pathogenesis in the early stage of bacterial infection.
\end{abstract}

\section{Background}

Genus Acinetobacter are important opportunistic pathogens in hospital-acquired infections [1-4]. They cause various types of human infections, including pneumonia, wound infections, urinary tract infections, bacteremia, and meningitis. Of the currently known 31 Acinetobacter species $[1,2,5]$, Acinetobacter baumannii is the most prevalent in clinical specimens. Numerous outbreaks caused by A. baumannii have been reported, which are of great concern in clinical settings $[6,7]$. Despite convincing evidence linking A. baumannii with human infections, the study of its pathogenic mechanism is still in its elementary stage.
Adherence of bacteria to epithelial cells is an essential step towards colonization and infection [8]. Bacterial adherence to host cells is mediated by fimbria or membrane components. Furthermore, many pathogenic bacteria are capable of invading non-phagocytic cells and evolve to survive within the host cells. The cellular invasion of bacteria contributes to evasion of humoral immunity, persistence in the host, and penetration into deep tissues. Bacterial pathogens gain entry to non-phagocytic cells via two mechanisms; a zipper-like mechanism and a trigger mechanism, which were initially classified based on morphological differences $[9,10]$. The zipper-like mechanism 
(receptor-mediated entry) requires the direct interaction of bacterial ligands to the host's cell surface receptors and involves local cytoskeletal rearrangement at the invasion site. In contrast, the trigger mechanism is initiated by the injected bacterial effector proteins delivered by the type III secretion system. The effector proteins regulate cytoskeleton dynamics and induce dramatic cytoskeletal rearrangements such as membrane ruffles. The previous study demonstrated that adherence of A. baumannii to human bronchial epithelial cells was mediated by fimbrial-like structures and entrapment of bacteria by cellular protrusions [11]. However, the mechanisms underlying invasion of A. baumannii in epithelial cells have not been explored so far.

Outer membrane proteins (Omps) of Gram-negative bacteria are key players in bacterial pathogenesis. OmpA of Escherichia coli, OspC of Borrelia burgdorferi, and Opa and OpcA of Neisseria meningitidis facilitate adherence to and invasion of bacteria in host cells [12-15]. OmpA of A. baumannii (AbOmpA) is the most abundant surface protein with a molecular mass of $38 \mathrm{kDa}$ and plays a role in permeability of small solutes. AbOmpA purified from A. baumannii ATCC $19606^{\mathrm{T}}$ and recombinant AbOmpA (rAbOmpA) bind to the surface of host cells and are localized in both the mitochondria and nuclei, which induce the death of host cells $[16,17]$. Based on previous findings that AbOmpA bound to and entered host cells, we hypothesized that AbOmpA is responsible for adherence to and invasion of A. baumannii in epithelial cells during the colonization and early stage of bacterial infection. In the present study, we investigated the potential of A. baumannii to invade epithelial cells and studied the role of AbOmpA in interactions of A. baumannii with epithelial cells.

\section{Results}

\section{A. baumannii invades epithelial cells dependently on both} the bacterial strains and cell types

To determine whether A. baumannii invaded epithelial cells, A. baumannii ATCC $19606^{\mathrm{T}}$ and four gentamicin-susceptible A. baumannii isolates from clinical specimens were selected and gentamicin protection assay was performed. A multiplicity of infection (MOI) of 100 that was previously optimized in the adherence assay of A. baumannii was used in the cell invasion assay [11]. To optimize infection time, human bronchial NCI-H292 cells were infected for up to $7 \mathrm{~h}$ with A. baumannii ATCC $19606^{\mathrm{T}}$. Intracellular bacterial counts increased steadily with incubation times (Fig. 1A), but cell death and destruction of cell monolayer appeared $5 \mathrm{~h}$ after infection. Therefore, we chose $5 \mathrm{~h}$ as the maximum infection period in the following study to exclude any secondary effect of cell death.
NCI-H292 cells were infected with four clinical A. baumannii isolates at an MOI of 100 for $5 \mathrm{~h}$. Cell invasion of A. baumannii was different between bacterial strains: A. baumannii 05KA103 was the most invasive $(7,581 \pm 2,365$ cfu), whereas A. baumannii 05P447 was the least invasive $(151 \pm 105 \mathrm{cfu})$ (Fig. 1B). To determine whether the cell invasion of A. baumannii is dependent on epithelial cell types, human bronchial NCI-H292 cells, human laryngeal HEp-2 cells, and human cervical HeLa cells were infected with A. baumannii strains. NCI-H292 and HEp-2 cells derived from the respiratory tract were more susceptible to cell invasion of $A$. baumannii than non-respiratory tractderived HeLa cells (Fig. 1B). Confocal microscopic images provided direct evidence of intracellular localization of $A$. baumannii (Fig. 1C and 1D).

\section{A. baumannii invades epithelial cells by a zipper-like mechanism}

To investigate the involvement of microfilaments and microtubules in the cell invasion of A. baumannii, epithelial cells were pretreated with cytoskeleton inhibitors, cytochalasin D for microfilaments and vinblastine for microtubules, and a gentamicin protection assay was performed. Both cytochalasin D and vinblastine significantly inhibited the cell invasion of A. baumannii. Pretreatment of NCI-H292 cells with cytochalasin D resulted in a decrease of intracellular bacteria of $93 \%$ and $90 \%$ in $A$. baumannii 05KA103 and type strain, respectively $(P<$ 0.005) (Fig. 2A). Cell invasion was also significantly decreased by vinblastine $(P<0.01)$ (Fig. $2 \mathrm{~B})$. These results suggest that $A$. baumannii invades epithelial cells through both microfilament- and microtubule-dependent uptake mechanisms.

The association of A. baumannii with epithelial cells was characterized in detail by electron microscopy. Scanning electron microscopy (SEM) exhibited the intimate interactions of A. baumannii with epithelial cells (Fig. 3A and $3 \mathrm{~B})$. The cell membrane extended to and wrapped around bacteria, but the membrane ruffles were not appeared. Transmission electron microscopy (TEM) illuminated the sequential cell invasion of $A$. baumannii. Bacteria were loosely attached to the cell surface and small membrane process protruded from the cells (Fig. 3C). After internalization of A. baumannii in epithelial cells, the cell membrane closed at the invasion site (Fig. 3D). Internalized bacteria were located in the membrane-bound vacuoles (Fig. 3E). There was no sign of a membrane ruffle in SEM and TEM images. Cellular changes, such as membrane wrapping around bacteria and fitted vacuoles to bacterial size, suggest that $A$. baumannii is internalized by a zipperlike mechanism, but not a trigger mechanism. 
A

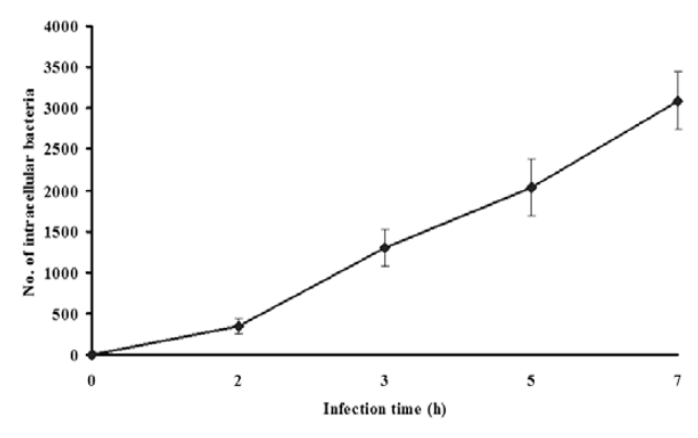

${ }_{\text {Atiin }}^{\text {ind }}$

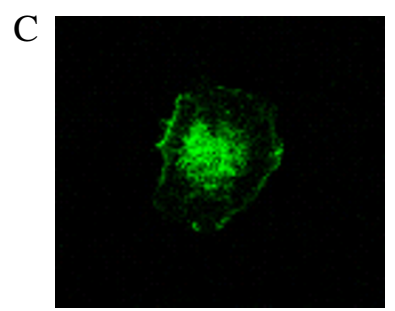

$\mathrm{D}$

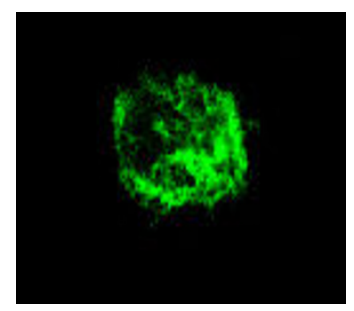

B

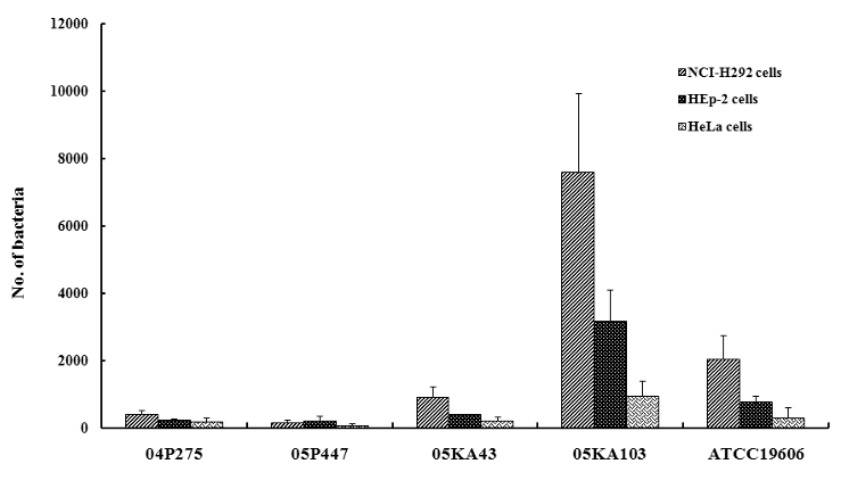

Bacteria

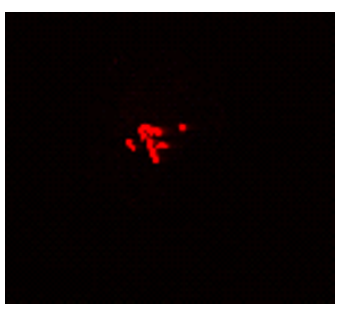

Merged
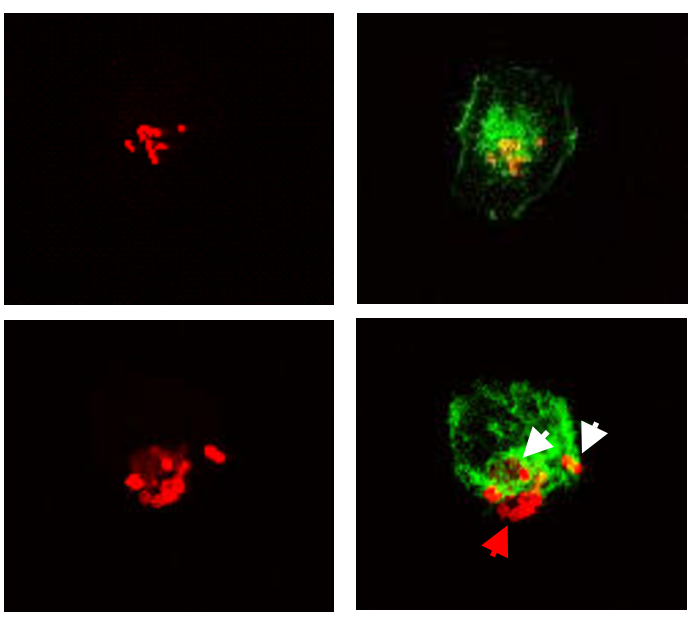

Figure I

Invasion of $A$. baumannii in epithelial cells. (A) $\mathrm{NCl}-\mathrm{H} 292$ cells were infected with $A$. baumannii ATCC I9606 ${ }^{\top}$ at an $\mathrm{MOI}$ of 100 up to $7 \mathrm{~h}$. The colony-forming units were enumerated to measure the time-course of invasion. The result represents the mean \pm standard deviation in duplicate wells and repeated a minimum of three separate times on separate days. (B) $\mathrm{NCl}$ $\mathrm{H} 292$, HEp-2, and HeLa cells were infected with A. baumannii strains at an MOl of 100 for 5 h. (C) $\mathrm{NCl}-\mathrm{H} 292 \mathrm{cells}$ were infected with A. baumannii 05KAI03 at an MOI of 100 for 5 h. Actin was stained with Alexa Fluor ${ }^{\circledR} 488$ phalloidin (green). Bacteria were stained with polyclonal anti-rabbit AbOmpA antibody, followed by a secondary antibody Alexa Fluor ${ }^{\circledR} 568$ (red). The analytical sectioning was performed from the top to the bottom of the cells. The figure represents a single section of the cells. (D). NCl-H292 cells were infected with A. baumannii ATCC 19606' at an MOI of 100 for 5 h. Actin filaments have wraparound-bacteria (white arrow). Red arrow indicates the extracellular bacteria. The figure represents all projection of sections in one picture.

\section{AbOmpA mediates the interaction of A. baumannii with epithelial cells}

To determine the potential interaction of AbOmpA with epithelial cells, the binding of rAbOmpA to various types of epithelial cells was analyzed by using the flow cytometry. It was found that rAbOmpA specifically bound to the surface of epithelial cells tested (Fig. 4). The mean fluorescence intensities of NCI-H292 and HEp-2 cells were higher than those of HeLa cells, which was in accordance with the cell invasion of A. baumannii. We determined whether rAbOmpA inhibited the interactions of $A$. bau- mannii with epithelial cells. The cytotoxicity of rAbOmpA was first assessed, because $\geq 6 \mu \mathrm{g} / \mathrm{ml}$ of AbOmpA purified from A. baumannii ATCC $19606^{\mathrm{T}}$ induced the cytotoxicity of HEp-2 cells [16]. However, $\leq 10 \mu \mathrm{g} / \mathrm{ml}$ of rAbOmpA did not induce any morphological and biochemical changes of epithelial cells for $5 \mathrm{~h}$ of incubation (data not shown). NCI-H292 and HEp-2 cells were pretreated with $10 \mu \mathrm{g} / \mathrm{ml}$ of rAbOmpA for $1 \mathrm{~h}$ and infected with the highly invasive A. baumannii 05KA103 for $1 \mathrm{~h}$. As a result, rAbOmpA attenuated the binding of $A$. baumannii to epithelial cells (Fig. 5A). Adherence of A. baumannii 05KA103 
A

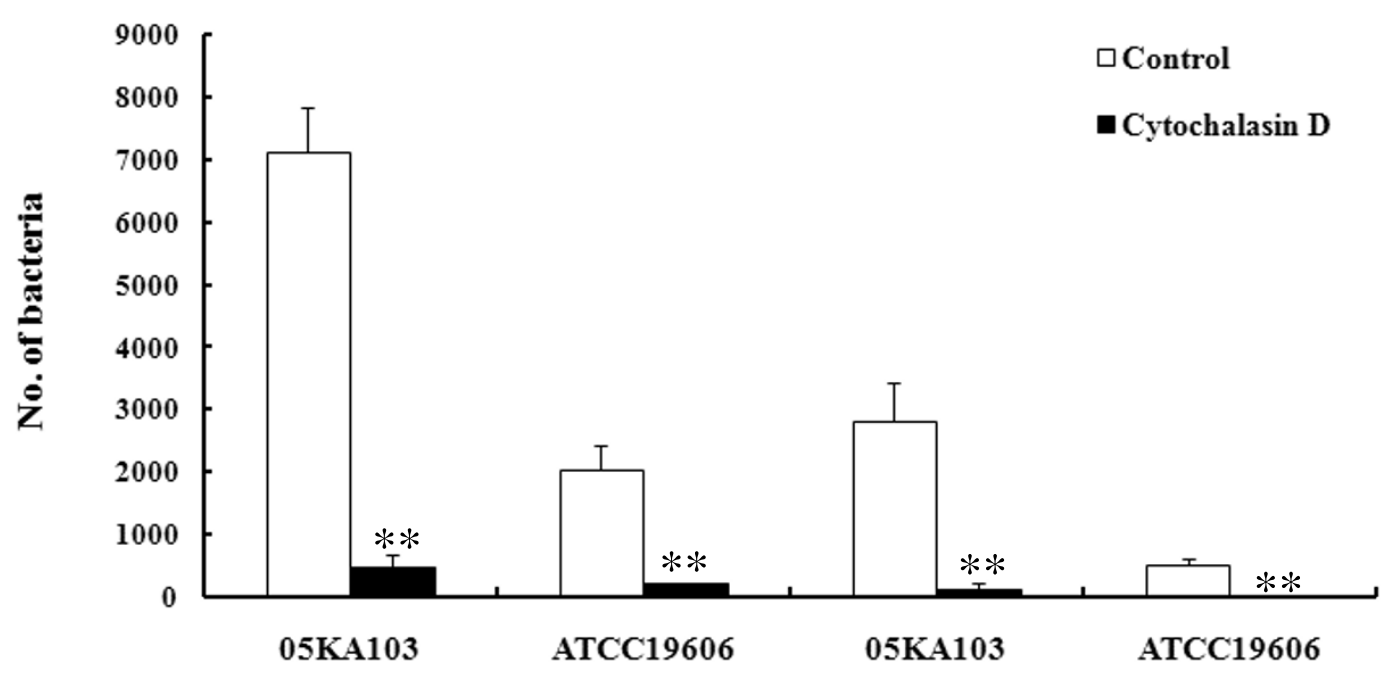

B

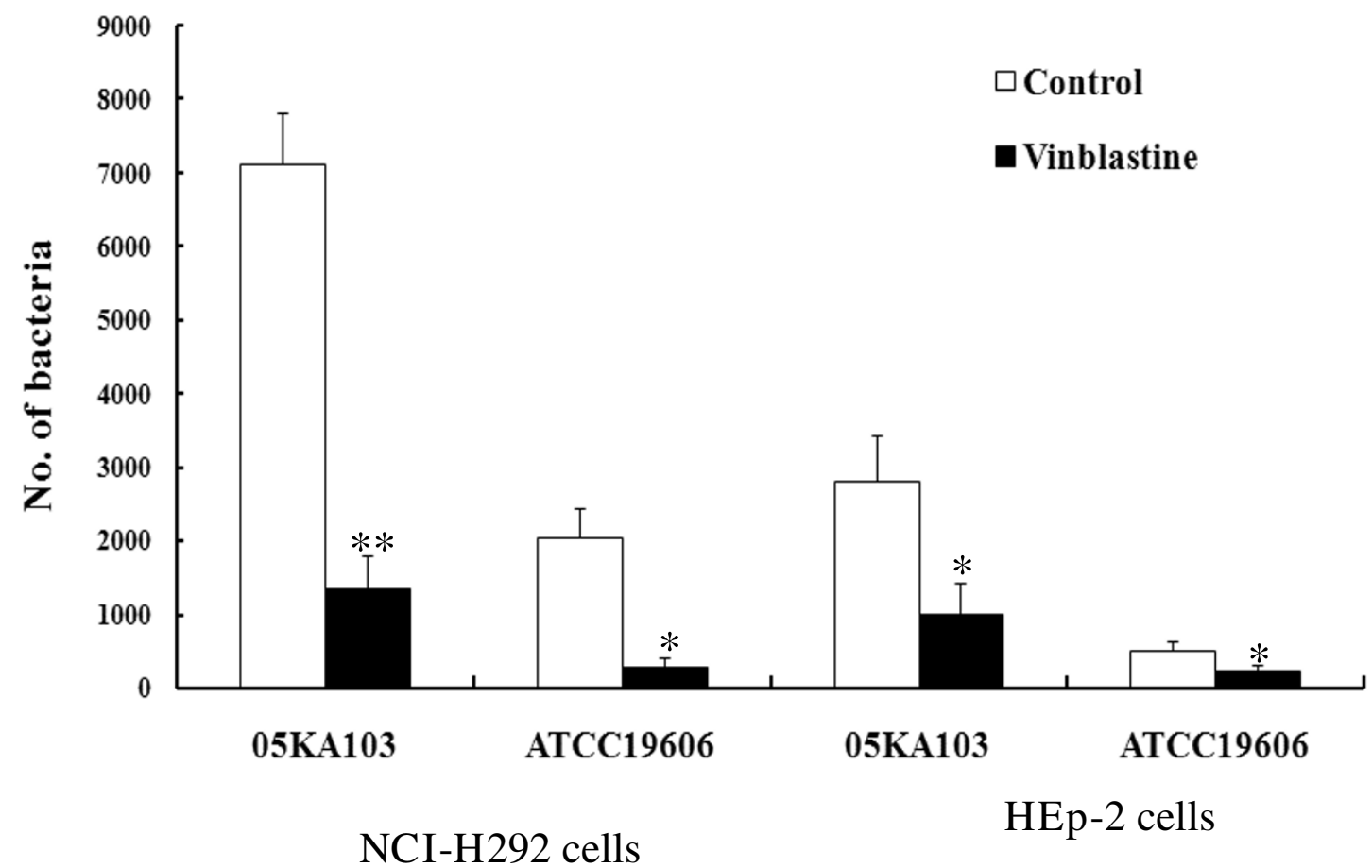

Figure 2

Inhibition of A. baumannii invasion by cytoskeleton inhibitors, cytochalasin D (A) and vinblastine (B). NCl-H292 and HEp-2 cells were pretreated with cytochalasin D $(2 \mu \mathrm{M})$ or vinblastine $(100 \mu \mathrm{M})$ for 30 min and maintained for $5 \mathrm{~h}$ of the entire infection period. A. baumannii invasion was measured as the number of bacteria per well using the gentamicin protection assay. Data represent the mean \pm standard deviation of three separate experiments. $* P<0.01, * * P<0.005$. 

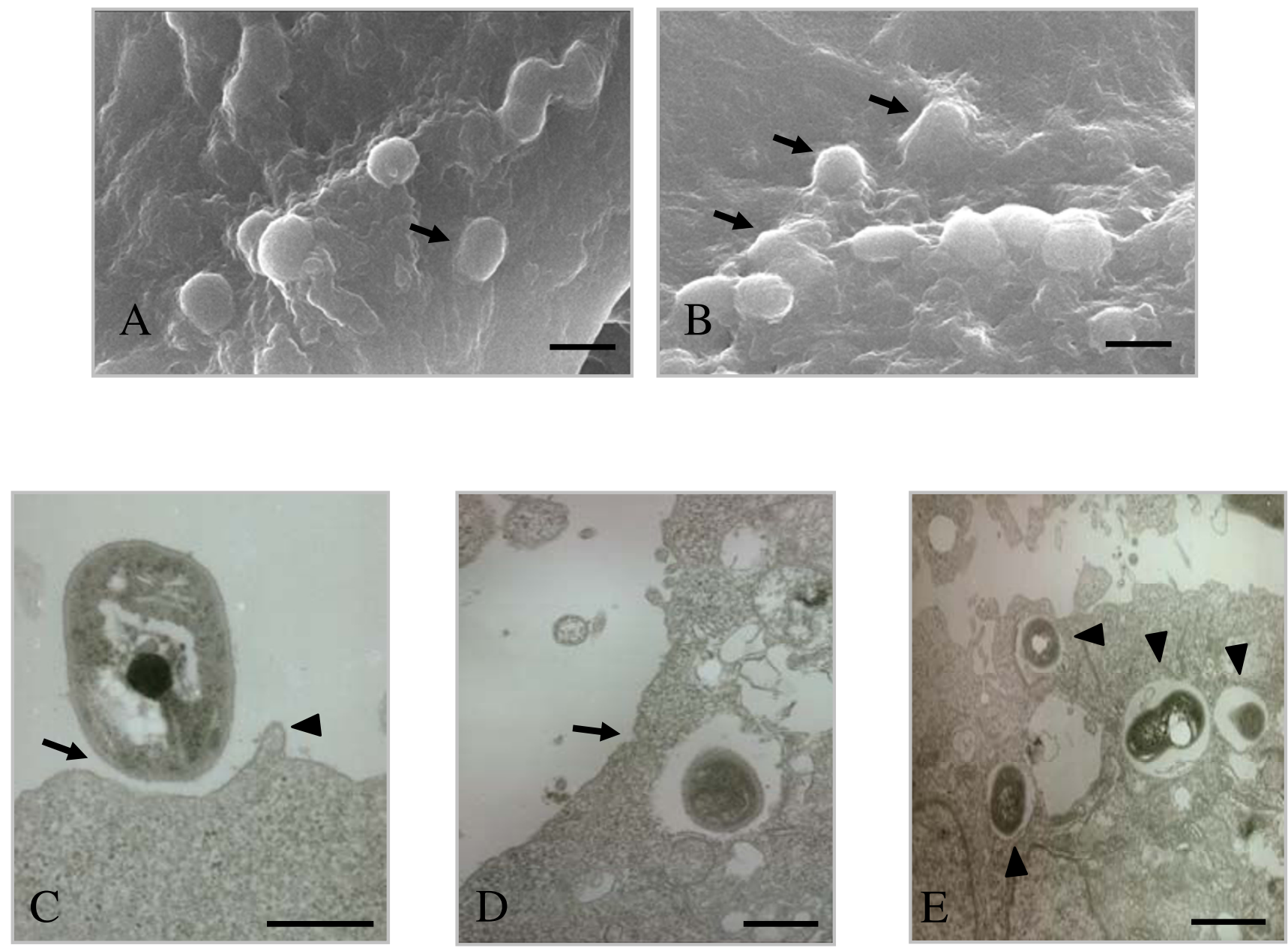

\section{Figure 3}

Electron micrographs demonstrating internalization of $A$. baumannii in epithelial cells. $\mathrm{NCl}-\mathrm{H} 292$ cells were infected with A. baumannii 05KAIO3 at an MOI of 100 for $5 \mathrm{~h}$. (A and B) SEM images showing interaction of $A$. baumannii with $\mathrm{NCl}-\mathrm{H} 292$ cells. The cell membrane was extended to and wrapped around A. baumannii (arrow). Bar represents $2 \mu \mathrm{m}$. (C to E) TEM images. (C) A. baumannii loosely anchored to the cell surface (arrow) and small protrusion from the cell was appeared (arrow head). (D) The cell membrane was fused after the bacterial internalization (arrow). (E) Intracellular bacteria were surrounded by a membrane-bound vacuole (arrow head). Bar represents I $\mu \mathrm{m}$.

to epithelial cells significantly decreased to $81 \%$ in HEp-2 cells $(P<0.001)$ and $87 \%$ in NCI-H292 cells $(P<0.001)$. To determine whether rAbOmpA also inhibited the cell invasion of $A$. baumannii, epithelial cells were pretreated with $10 \mu \mathrm{g} / \mathrm{ml}$ of rAbOmpA for $1 \mathrm{~h}$ and infected with $A$. baumannii for $5 \mathrm{~h}$. The cell invasion of A. baumannii 05 KA103 significantly decreased to $69 \%$ in NCI-H292 cells $(P<0.05)$ and $67 \%$ in HEp-2 cells $(P<0.05)$ (Fig. $5 \mathrm{~B})$. Collectively, these results suggest that AbOmpA mediates the adherence to and invasion of A. baumannii in epithelial cells.

\section{Cell invasion of isogenic AbOmpA- mutant decreases as compared with wild-type bacteria}

To compare the cell adherence of A. baumannii ATCC $19606^{\mathrm{T}}$ and its isogenic AbOmpA- mutant KS37, NCIH292 and HEp-2 cells were infected with bacteria for $1 \mathrm{~h}$. Wild-type bacteria exhibited dispersed adherence (Fig. $6 \mathrm{~A})$. The number of adherent bacteria was 1-3 bacteria/ infected cells and the percentage of infected cells was less than $3 \%$. The total number of adherent bacteria per 100 cells was $7.5 \pm 4.8$ in NCI-H292 cells. However, isogenic AbOmpA- mutant formed microcolonies on the surface of epithelial cells, rather than dispersed adherence. The number of adherent bacteria per 100 cells was not significantly different between wild-type bacteria and AbOmpA- 


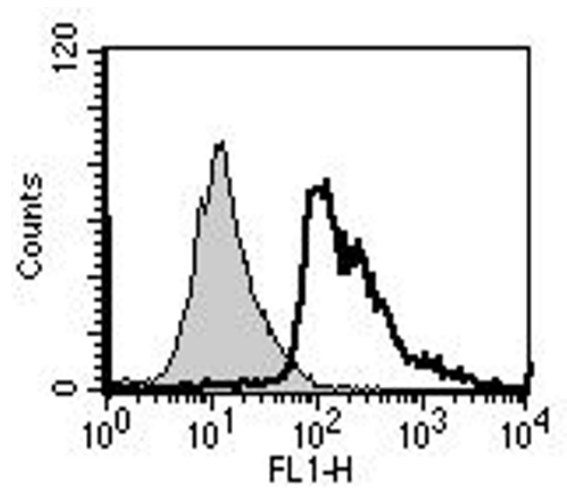

NCI-H292 cells

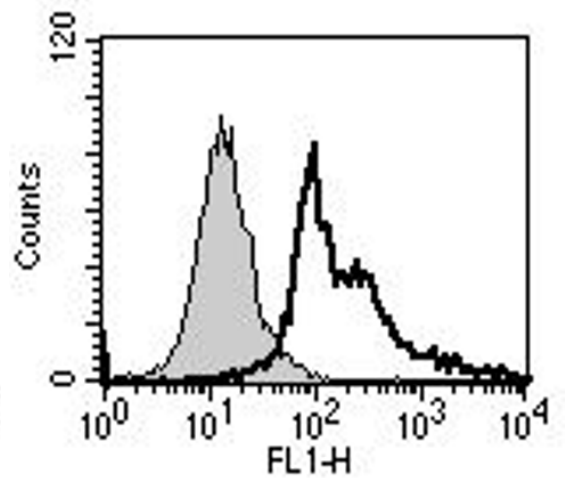

HEp-2 cells

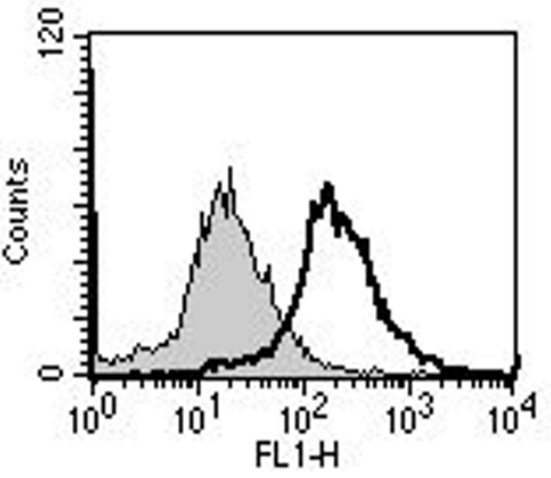

HeLa cells

Figure 4

Cell surface binding of rAbOmpA. The grey shaded region represents the control fluorescent level seen in the cells treated with polyclonal anti-rabbit AbOmpA antibody and Alexa Fluor ${ }^{\circledR} 488$-conjugated secondary antibody without the addition of rAbOmpA. The solid line represents the fluorescent level seen in the cells treated with $6 \mu \mathrm{g} / \mathrm{ml}$ of $\mathrm{rAbOmpA}$.

mutant. To assess the cell invasion of wild-type bacteria and AbOmpA- mutant, epithelial cells were infected with bacteria for $5 \mathrm{~h}$. The cell invasion of AbOmpA- mutant significantly decreased to $95 \%$ as compared with wild-type bacteria $(P<0.001)$ (Fig. 6B). A low cell invasion of AbOmpA- mutant was not due to retarded bacterial growth, because there was no difference in bacterial growth between wild-type bacteria and AbOmpA- mutant (data not shown).

\section{AbOmpA plays a role in A. baumannii pathogenesis in vivo}

To determine the role of AbOmpA on A. baumannii pathogenesis in vivo, the murine pneumonia model was introduced. The C57BL/6 mice were intratracheally infected with $1 \times 10^{9} \mathrm{cfu}$ of wild-type bacteria and isogenic AbOmpA- mutant, and euthanized two days after bacterial challenge. Total number of bacterial counts in the lungs were similar between mice infected with wild-type bacteria $\left(1.58 \times 10^{5} \mathrm{cfu} / \mathrm{g}\right)$ and $\mathrm{AbOmpA}-$ mutant $\left(6.75 \times 10^{4}\right.$ $\mathrm{cfu} / \mathrm{g})$, but lung histopathology such as the infiltration of polymorphonuclear leukocytes and destruction of alveolar structures was prominently observed in the mice infected with wild-type bacteria (data not shown). Bacteremia occurred in mice infected with both bacterial strains, but there was a significant difference in the bacterial burden between the mice infected with wild-type bacteria $\left(3.5 \times 10^{3} \mathrm{cfu} / \mathrm{ml}\right)$ and isogenic AbOmpA- mutant $(2.3 \times$ $10 \mathrm{cfu} / \mathrm{ml}$ ). These results suggest that AbOmpA is directly associated with the lung pathology and is responsible for the dissemination of $A$. baumannii into the bloodstream.

\section{Discussion}

It was clearly demonstrated that $A$. baumannii has the potential to invade epithelial cells. Host cells play a significant role in determining the cell invasion of A. baumannii. Epithelial cells derived from the respiratory tract were more susceptible to A. baumannii invasion than epithelial cells derived from non-respiratory tract. Moreover, the binding capacity of rAbOmpA in respiratory tract-derived epithelial cells was higher than that of non-respiratory tract-derived epithelial cells. These findings may be partly responsible for the prevalence of A. baumannii in the respiratory tract, which is the most frequent colonization and infection site. The efficiency of cell invasion of $A$. baumannii was lower than other invasive pathogens such as $E$. coli, Campylobacter upsaliensis, Helicobacter pylori, N. memingitidis, Pseudomonas aeruginosa, and Yersinia enterocolitica [18-21]. A previous study showed that most clinical $A$. baumannii isolates infected only $20-30 \%$ of NCI-H 292 cells and the number of adhering bacteria was 1-2 bacteria per infected cell [11]. This finding reflects the low cellular invasion of A. baumannii in the current study. A relatively low adherence and invasion of $A$. baumannii in epithelial cells may contribute to a low virulence of this opportunistic pathogen, which particularly infects critically ill or severely wounded patients $[1,2]$.

A. baumannii invaded epithelial cells by a zipper-like mechanism, as demonstrated by the morphological characteristics. A. baumannii was loosely attached to the surface of epithelial cells. This observation is consistent with the previous study regarding adherence of $A$. baumannii to NCI-H292 cells [11]. However, the entrapment of bacteria by cellular protrusions, as noted in the previous study, 
A

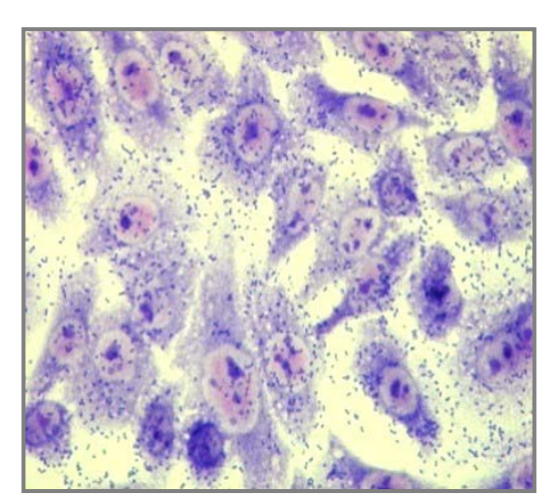

rAbOmpA + A. baumannii

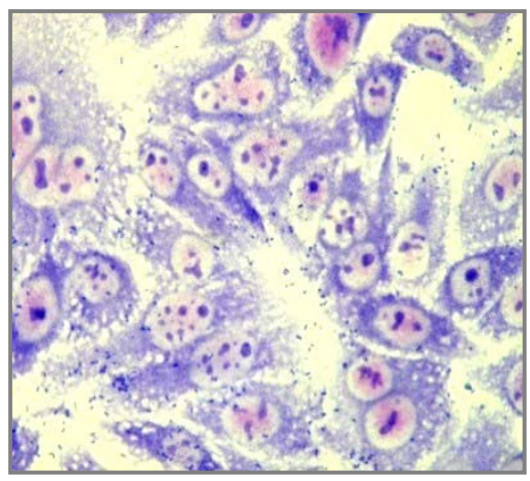

B

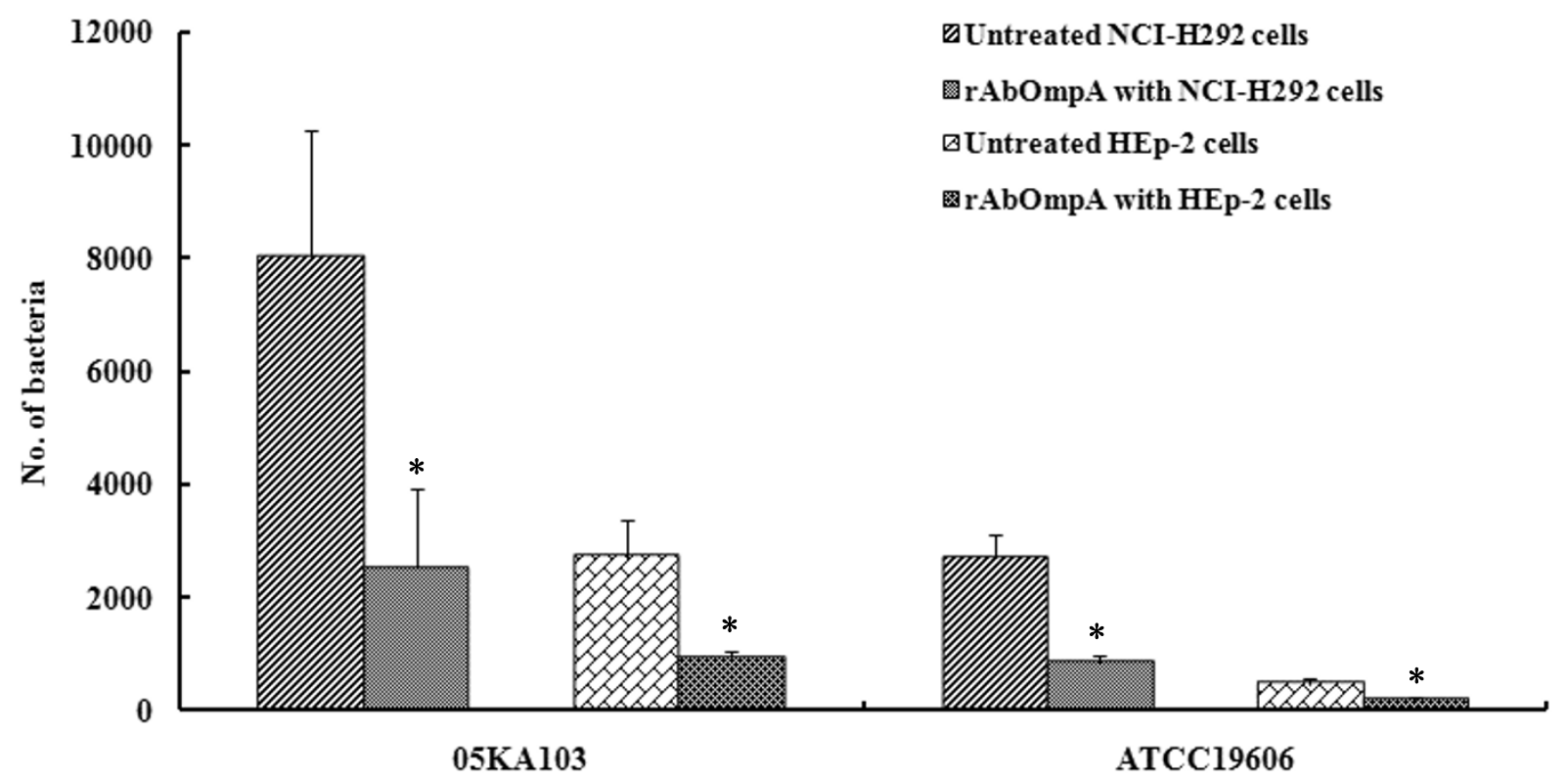

Figure 5

rAbOmpA attenuates the interaction of $A$. baumannii with epithelial cells. (A) $\mathrm{NCl}-\mathrm{H} 292$ cells were pretreated with $10 \mu \mathrm{g} / \mathrm{ml}$ of rAbOmpA for I $\mathrm{h}$ and then infected with A. baumannii $05 \mathrm{KA} 103$ at an MOI of 100 for I h. Cell monolayer was stained with the Giemsa solution. (B) NCl-H292 and HEp-2 cells were pretreated with $10 \mu \mathrm{g} / \mathrm{ml}$ of $\mathrm{rAbOmpA}$ for $\mathrm{I} \mathrm{h}$ and infected with A. baumannii at an MOI of 100 for $5 \mathrm{~h}$. Invasion efficiency was expressed as the number of bacteria per well using the gentamicin protection assay. The results represent the mean \pm standard deviation of three separate experiments. $* P<0.05$.

was not observed. After loose attachment of A. baumannii to epithelial cells, the cell membrane was extended to and wrapped around the bacteria, which are typical characteristics of the zipper-like mechanism. These cellular responses against $A$. baumannii were mediated by actin rearrangement and membrane reorganization. Confocal images and gentamicin protection assay with cytochalasin
D showed the involvement of actin filaments in the cell invasion of A. baumannii. The microtubule inhibitor vinblastine also significantly inhibited the cell invasion of $A$. baumannii. Vinblastine may inhibit the transport of membrane-bound bacteria from the plasma membrane to the cytoplasm by preventing their movement in the microtubule-mediated contractile process. Once inside the host 
A

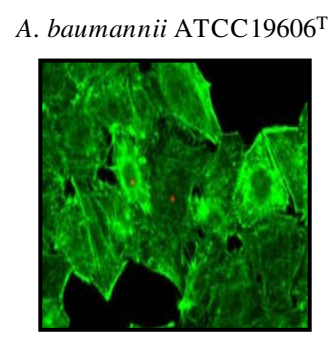

AbOmpA- mutant

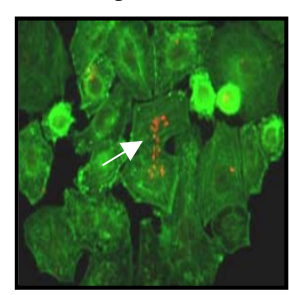

B

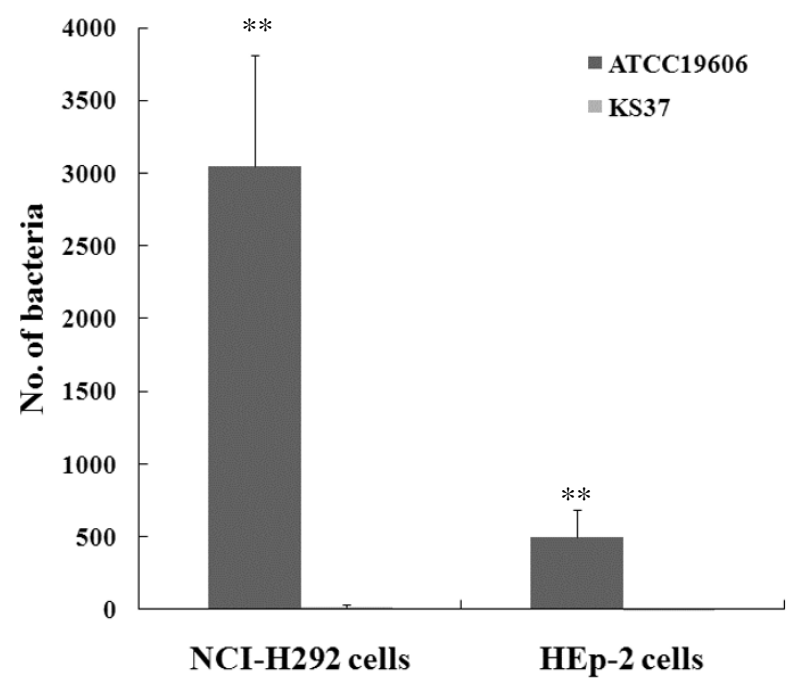

\begin{tabular}{ccc}
\hline \multirow{2}{*}{ Strain } & \multicolumn{2}{c}{ No. of Bacteria } \\
\cline { 2 - 3 } & NCI-H292 cells & HEp-2 cells \\
\hline ATCC 19606 & $3,051 \pm 757$ & $500 \pm 184$ \\
K S37 & $18 \pm 13$ & $2.5 \pm 0.5$ \\
\hline
\end{tabular}

\section{Figure 6}

Adherence and invasion of A. baumannii ATCC $19606^{\top}$ and isogenic AbOmpA- mutant in epithelial cells. (A). Adherence of $A$. baumannii to epithelial cells. $\mathrm{NCl}-\mathrm{H} 292$ cells were infected with $A$. baumannii strains at an $\mathrm{MOI}$ of $\mathrm{I} 00$ for I h. Actin was stained with Alexa Fluor ${ }^{\circledR} 488$ phalloidin (green). Bacteria were stained with polyclonal anti-rabbit AbOmpA antibody, followed by a secondary antibody Alexa Fluor ${ }^{\circledR} 568$ (red). (B). NCl-H292 and HEp-2 cells were infected with A. baumannii at an $\mathrm{MOI}$ of 100 for $5 \mathrm{~h}$. Invasion efficiency was expressed as the number of bacteria per well using the gentamicin protection assay. Results represent the mean and standard deviation of three separate experiments on separately days. $* P<$ 0.001 .

cells, internalized A. baumannii was located in the membrane-bound vacuoles. This result, in conjunction with the results of the gentamicin protection assay, suggests that A. baumannii survives within membrane-bound vacuoles in the cytoplasm. Many intracellular bacteria are located in a membrane-bound vacuole compartment [22], but some, such as Neisseria spp., Listeria monocytogenes, Salmonella spp., and Yersinia spp., escape the cytoplasmic vacuoles and are freely located in the cytoplasm [23-26]. In regards to most pathogenic bacteria, a membrane-bound vacuole containing pathogens does not undergo sequential fusion with endosomes or lysosomes for its own benefit. Further studies are needed to characterize the fate of intracellular A. baumannii to understand the survival and dissemination of bacteria.

Outer membrane proteins of Gram-negative bacteria are known to be directly involved in the interaction of bacteria with host cells. The present study focused on whether AbOmpA is responsible for the interaction of A. baumannii with epithelial cells. Our data suggest that AbOmpA is a major effector molecule of A. baumannii interacting with epithelial cells according to the following experimental results: (i) rAbOmpA directly binds to the surface of epithelial cells; (ii) pre-treatment of rAbOmpA significantly attenuates the binding of A. baumannii to epithelial cells; (iii) rAbOmpA significantly inhibits the cell invasion of $A$. baumannii, and (iv) the cell invasion of AbOmpA- mutant decreased to $95 \%$ in comparison to wild-type bacteria. In a murine pneumonia model, wild-type bacteria and isogenic AbOmpA- mutant induced different lung pathologies. Moreover, wild-type A. baumannii had the capacity to disseminate into the bloodstream, whereas AbOmpAmutant rarely cross barriers to disseminate in this manner. Accordingly, AbOmpA plays an important role in the A. baumannii pathogenesis regarding the induction of pneumonia and bacteremia.

A. baumannii can adhere to and invade epithelial cells during colonization and the early stage of infection. In addition to its biological role as a porin, AbOmpA plays a versatile role in the $A$. baumannii pathogenesis regarding 
its interaction with epithelial cells, the induction of apoptosis of host cells, and the dissemination of bacteria into the bloodstream. Our data provide a novel insight into $A$. baumannii pathogenesis and contribute to further our knowledge of A. baumannii infections.

\section{Conclusion}

A. baumannii invades epithelial cells depending on both bacterial strains and cell types. Cellular invasion is mediated by both microfilament- and microtubule-dependent uptake mechanisms. AbOmpA is a microbial component responsible for the adherence to and invasion of $A$. baumannii in epithelial cells. The data obtained provide a novel insight into the A. baumannii pathogenesis in the early stage of bacterial infection.

\section{Methods}

\section{Bacterial strains}

A. baumannii ATCC $19606^{\mathrm{T}}$ and its isogenic AbOmpAKS37 mutant [16] were used in this study. Four clinical A. baumannii isolates were obtained from hospitalized patients: 05KA43 and 05P447 from blood, 05KA103 from cerebrospinal fluid, and 04P275 from wounds. All A. baumannii strains were susceptible to gentamicin. The genus Acinetobacter was identified by phenotypic markers and species identification was confirmed by amplified ribosomal DNA restriction analysis [27]. For the adherence and invasion assays, A. baumannii strains were grown on blood agar plates at $37^{\circ} \mathrm{C}$ for $18 \mathrm{~h}$ and bacterial cells were suspended in a cell culture medium without antibiotics at a density of $1.0 \times 10^{8} \mathrm{cfu} / \mathrm{ml}$.

\section{Cell cultures}

Human laryngeal epithelial cells (HEp-2, CCL-23) and cervical carcinoma cells (HeLa, CCL-2) were cultured in Dulbecco's modified Eagle's medium supplemented with $2 \mathrm{mM}$ L-glutamine, $1000 \mathrm{U}$ of penicillin $\mathrm{G}$ per $\mathrm{ml}, 50 \mu \mathrm{g}$ of streptomycin per $\mathrm{ml}$, and $10 \%$ fetal bovine serum. Human bronchial epithelial cells (NCI-H292, CRL-1848) were cultured in a RPMI 1640 medium containing the above supplements. The cells were maintained at $37^{\circ} \mathrm{C}$ in $5 \% \mathrm{CO}_{2}$. Confluent growth was obtained in $100 \mathrm{~mm}$ diameter dishes. All cell culture media and supplements were purchased from HyClone Laboratories Inc.

\section{Cloning and purification of rAbOmpA}

The cloning of the ompA gene and purification of recombinant AbOmpA (rAbOmpA) were performed as described previously [28]. In brief, the ompA gene $(1,317$ bp) of A. baumannii ATCC $19606^{\mathrm{T}}$ was amplified by PCR using the upstream primer (5'-ACAGGATCCATGAAATTGAGTCGTATT-3') and downstream primer (5'ACAAGCTTTTATTGAGCTGCTGCA-3'). PCR products were ligated into the pET28a expression vector (Novagen). E. coli BL21 (DE3)/pET28a carrying the ompA gene were grown in Luria-Bertani (LB) broth at $37^{\circ} \mathrm{C}$ and rAbOmpA was overexpressed with $1 \mathrm{mM}$ IPTG at $25^{\circ} \mathrm{C}$ for 4 h. After bacterial sonication, the supernatant containing the soluble form of rAbOmpA was collected and loaded on a $5 \mathrm{ml} \mathrm{HiTrap}{ }^{\mathrm{TM}}$ FF column (Amersham Biosciences). His-tagged rAbOmpA was eluted by elution buffer (20 $\mathrm{mM}$ sodium phosphate, $500 \mathrm{mM} \mathrm{NaCl}$, and $500 \mathrm{mM}$ imidazole, pH 7.4). The rAbOmpA samples were dialyzed against the elution buffer without imidazole and phosphate-buffered saline (PBS). The rAbOmpA samples were mixed with polymyxin B-agarose (St. Louis. Mo, USA) to remove endotoxin complex. The samples were concentrated by Centricon (2,000 MW cut off; Millipore) and stored at $-70^{\circ} \mathrm{C}$.

\section{Antibodies}

Polyclonal anti-AbOmpA antiserum against the purified OmpA from A. baumannii ATCC $19606^{\mathrm{T}}$ was raised in rabbits by routine immunogenic procedures [29].

\section{Bacterial adherence assay}

In each well of a 24 well cell culture plates, A. baumannii was added to a monolayer of epithelial cells at a ratio of bacteria to host cells of 100:1 (MOI of 100). The cells infected with bacteria were incubated in a $5 \% \mathrm{CO}_{2}$ at $37^{\circ} \mathrm{C}$ for $1 \mathrm{~h}$. The cells were washed five times with PBS, fixed with methanol for $20 \mathrm{~min}$, and stained with Giemsa solution [11].

\section{Bacterial invasion assay}

Epithelial cells grown in 24 well plates were infected with A. baumannii for the indicated times at an MOI of 100 . Culture media were removed and the cell monolayer was washed three times with PBS. A fresh culture medium containing $300 \mu \mathrm{g} / \mathrm{ml}$ of gentamicin was added and incubated for another $2 \mathrm{~h}$. The cells were washed five times with PBS and lysed with $0.1 \%$ Triton X-100 at $37^{\circ} \mathrm{C}$ for 20 min. Dilutions from each well were plated on nutrient agar and colonies were enumerated $20 \mathrm{~h}$ after incubation. The invasion efficiency was calculated as the average number of bacteria per well. Each invasion assay was performed in duplicate and repeated on a minimum of three separate days. To determine the effect of cytoskeleton inhibitors on A. baumannii invasion, $2 \mu \mathrm{M}$ of cytochalasin $\mathrm{D}$ (Sigma) or $100 \mu \mathrm{M}$ of vinblastine (Sigma) was added to the cells 30 min before the addition of bacteria and maintained in the medium for the entire infection period. Neither cytoskeletal inhibitors affected the bacterial viability and cellular viability at the concentrations used. To determine the inhibitory effect of AbOmpA on bacterial invasion, epithelial cells were pretreated with $10 \mu \mathrm{g} / \mathrm{ml}$ of rAbOmpA $1 \mathrm{~h}$ before bacteria infection. 


\section{Cell surface binding of rAbOmpA}

Epithelial cells were grown in $100 \mathrm{~mm}$ dishes and then detached from the dishes by treatment with $0.5 \mathrm{M}$ EDTA solution. The cells were washed three times and suspended at $5 \times 10^{5}$ cells $/ \mathrm{ml}$ in PBS. The cells were incubated in the presence of polyclonal anti-rabbit AbOmpA antibody $(1: 1,000)$. After washing, the cells were incubated with $6 \mu \mathrm{g} / \mathrm{ml}$ of rAbOmpA at $4^{\circ} \mathrm{C}$ for $20 \mathrm{~min}$ and washed three times in cold PBS. The samples were then incubated with anti-rabbit AbOmpA antibody $(1: 1,000)$ on ice for 1 h. The cells were washed three times with cold PBS and incubated with Alexa Fluor ${ }^{\circledast} 488$-conjugated anti-rabbit IgG (1:500) on ice for $1 \mathrm{~h}$. The labelled cells were analyzed by flow cytometry (Becton Dickinson).

\section{Fluorescence and confocal microscopy}

NCI-H292 cells were grown on $13 \mathrm{~mm}$ diameter glass coverslips at $2 \times 10^{5}$ per coverslip. A. baumannii was infected at an MOI of 100 and incubated at $37^{\circ} \mathrm{C}$ for $5 \mathrm{~h}$. The coverslips were washed with PBS and fixed with $3.7 \%$ paraformaldehyde for $30 \mathrm{~min}$. Cells were permeabilized with a PBS containing $0.25 \%$ Triton X-100 for 10 min. Actin was stained with Alexa Fluor ${ }^{\circledR} 488$ phalloidin (Molecular Probes). A. baumannii was labeled with polyclonal antirabbit AbOmpA antibody $(1: 1,000)$, followed by Alexa Fluor ${ }^{\boxplus 68}$-conjugated goat anti-rabbit IgG antibody (Molecular Probes). The association of A. baumannii with epithelial cells was observed either with a Nikon inverted fluorescence microscope or Leica confocal microscope.

\section{TEM and SEM}

For TEM, NCI-H292 cells were grown in $35 \mathrm{~mm}$ culture dishes at a concentration of $1 \times 10^{6}$ cells/well. A. baumannii was infected to the monolayer of NCI-H292 cells at an MOI of 100 for $5 \mathrm{~h}$. The cells were washed five times with PBS and harvested by using trypsin-EDTA $(500 \mathrm{mg} / \mathrm{ml}$ trypsin, $200 \mathrm{mg} / \mathrm{ml}$ EDTA). The cells were centrifuged at $300 \mathrm{~g}$ for $5 \mathrm{~min}$ and washed with PBS. The cells were resuspended in a fixative (4\% paraformaldehyde and 1\% glutaraldehyde, $\mathrm{pH}$ 7.0). The samples were then incubated in osmium tetroxide, gradually dehydrated in a series of ethanol and embedded in Epon. Thin sections were prepared by using an ultramicrotome with a diamond knife. The samples were examined by a Hitachi 7000 transmission electron microscope. For SEM, NCI$\mathrm{H} 292$ cells were grown on $13 \mathrm{~mm}$ diameter glass coverslips at a concentration of $2 \times 10^{5}$ cells/well and bacteria were infected at an MOI of 100 for $5 \mathrm{~h}$. The samples were washed with PBS and fixed with $2.5 \%$ glutaraldehyde at room temperature. The cells were gradually dehydrated in a series of ethanol and dried to a critical point. The samples were then coated with a layer of gold and examined under $15 \mathrm{kV}$ accelerating voltage in a Hitachi S-4300 field emission scanning electron microscope.

\section{Animal experiments}

Six-week-old female C57BL/6 mice were maintained under specific-pathogen-free conditions. For the pneumonia model, the mice were anesthetized with pentobarbital and then $100 \mu \mathrm{l}$ of $1 \times 10^{10} \mathrm{cfu} / \mathrm{ml}$ of bacteria were administrated intratracheally. Groups containing three mice were infected with wild-type A. baumannii and isogenic AbOmpA- mutant. The control mice groups were injected with $100 \mu \mathrm{l}$ of PBS. The animals were suspended in a supine position on a $60^{\circ}$ incline board. Their trachea was exposed surgically and bacteria were introduced intratracheally via a syringe with a $301 / 2$ gauged-needle. The incisions were closed by surgical sutures. The bacterial density of each run was confirmed by serial dilution and culture of an aliquot from each inoculum. The lungs of mice infected with bacteria were removed two days after bacterial challenge. For histological analysis, tissues were stained with hematoxylin/eosin and observed by using a Nikon microscope. For bacterial counts, lungs were homogenized by using a glass syringe piston and then serial 10-fold suspension was inoculated onto MacConkey agar plates (Difco Laboratories). Blood was directly plated on a MacConkey agar plate.

\section{Statistical analysis}

The statistical significance of the data was determined by the Student's $t$-test. A $P$ value of $<0.05$ was considered to be statistically significant.

\section{Abbreviations}

AbOmpA: Outer membrane protein A of A. baumannii; PBS: phosphate-buffered saline; LB broth: Luria-Bertani broth.

\section{Authors' contributions}

$\mathrm{CH}$ designed this study, carried out all experimental work, and completed the data analysis. IS performed the FACS experiments. YC participated in the data analysis and evaluation of the results. TI contributed to the electron microscopy experiments. JC coordinated the study, assisted in writing the manuscript, and revised the final manuscript. All authors read and approved the manuscript.

\section{Acknowledgements}

This study was supported by the Korea Research Foundation Grant funded by the Korean Government (MOEHRD, Basic Research Promotion Fund) (KRF-2006-3 I2-E00070).

\section{References}

I. Dijkshoorn L, Nemec A, Seifert H: An increasing threat in hospitals: multidrug-resistant Acinetobacter baumannii. Nat Rev Microbiol 2007, 5:939-95I.

2. Peleg AY, Seifert H, Paterson DL: Acinetobacter baumannii: emergence of a successful pathogen. Clin Microbiol Rev 2008, 2l:538-582.

3. Go ES, Urban C, Burns J, Kreiswirth B, Eisner W, Mariano N, Mosinka-Snipas K, Rahal J]: Clinical and molecular epidemiology 
of Acinetobacter infections sensitive only to polymyxin B and sulbactam. Lancet 1994, 344: 1329-1332.

4. Seifert H, Strate A, Schulze A, Pulverer G: Bacteremia due to Acinetobacter species other than Acinetobacter baumannii. Infection 1994, 22:379-385.

5. Nemec A, Dijkshoorn L, Cleenwerck I, De Baere T, Janssens D, Reijden TJ van der, Jezek P, Vaneechoutte M: Acinetobacter parvus sp. Nov., a small-colony-forming species isolated from human clinical specimens. Int J Sys Evol Microbiol 2003, 53:I563-I567.

6. Fournier PE, Richet $\mathrm{H}$ : The epidemiology and control of Acinetobacter baumannii in health care facilities. Clin Infect Dis 2006, 42:692-699.

7. Naiemi NA, Duim B, Savelkoul PH, Spanjaard L, de Jonge E, Bart A, Vandenbroucke-Grauls CM, de Jong MD: Widespread transfer of resistance genes between bacterial species in an intensive care unit: implications for hospital epidemiology. J Clin Microbiol 2005, 43:4862-4864.

8. Beachey EH: Adhesin-receptor interactions mediating the attachment of bacteria to mucosal surfaces. J Infect Dis 1981, 1 43:325-345.

9. Alonso A, García-del Portillo F: Hijacking of eukaryotic functions by intracellular bacterial pathogens. Int Microbiol 2004, 7:181-191.

10. Dramsi $\mathrm{S}$, Cossart $\mathrm{P}$ : Intracellular pathogens and the actin cytoskeleton. Annu Rev Cell Dev Biol 1998, 14:137-166.

II. Lee JC, Koerten H, Broek P van den, Beekhuizen H, Wolterbeek R, Barselaar M van den, Reijden T Van der, Meer J Van der, Gevel J Van de, Dijkshoorn L: Adherence of Acinetobacter baumannii strains to human bronchial epithelial cells. Res Microbiol 2006, I 57:360-366.

12. Khan NA, Shin S, Chung JW, Kim KJ, Elliott S, Wang Y, Kim KS: Outer membrane protein $A$ and cytotoxic necrotizing factor-I use diverse signaling mechanisms for Escherichia coli KI invasion of human brain microvascular endothelial cells. Microb Pathog 2003, 35:35-42.

13. Moore J, Bailey SE, Benmechernene Z, Tzitzilonis C, Griffiths NJ, Virji M, Derrick JP: Recognition of saccharides by the OpcA, OpaD, and OpaB outer membrane proteins from Neisseria meningitides. J Biol Chem 2005, 280:3 |489-3। 497.

14. Pal U, Yang X, Chen M, Bockenstedt LK, Anderson JF, Flavell RA, Norgard MV, Fikrig E: OspC facilitates Borrelia burgdorferi invasion of Ixodes scapularis salivary glands. I Clin Invest 2004, I I 3:220-230.

15. Prasadarao NV, Wass CA, Weiser JN, Stins MF, Huang SH, Kim KS: Outer membrane protein A of Escherichia coli contributes to invasion of brain microvascular endothelial cells. Infect Immun 1996, 64:146-153.

16. Choi CH, Lee EY, Lee YC, Park TI, Kim HJ, Hyun SH, Kim SA, Lee SK, Lee JC: Outer membrane protein 38 of Acinetobacter baumannii localizes to the mitochondria and induces apoptosis of epithelial cells. Cell Microbiol 2005, 7: I I 27-I I 38.

17. Choi CH, Hyun SH, Lee JY, Lee JS, Lee YS, Kim SA, Chae JP, Yoo SM, Lee JC: Acinetobacter baumannii outer membrane protein A targets the nucleus and induces cytotoxicity. Cell Microbiol 2008, 10:309-319.

18. Capecchi B, Adu-Bobie J, Di Marcello F, Ciucchi L, Masignani V, Taddei A, Rappuoli R, Pizza M, Arico B: Neisseria meningitidis NadA is a new invasin which promotes bacterial adhesion to and penetration into human epithelial cells. Mol Microbiol 2005, 55:687-698.

19. Fleiszig SM, Zaidi TS, Pier GB: Pseudomonas aeruginosa invasion of and multiplication within corneal epithelial cells in vitro. Infect Immun 1995, 63:4072-4077.

20. Kwok T, Backert S, Schwarz S, Berger J, Meyer TF: Specific entry of Helicobacter pylori into cultured gastric epithelial cells via a zipper-like mechanism. Infect Immun 2002, 70:2 I08-2 I20.

21. Mooney A, Byrne C, Clyne M, Johnson-Henry K, Sherman P, Bourke $B$ : Invasion of human epithelial cells by Campylobacter upsaliensis. Cell Microbiol 2003, 5:835-847.

22. Sinai AP, Joiner KA: Safe haven: the cell biology of nonfusogenic pathogen vacuoles. Annu Rev Microbiol 1997, 51:415-462.

23. Isberg RR: Discrimination between intracellular uptake and surface adhesion of bacterial pathogens. Science 199I, 252:934-938.

24. Jarvis GA, Li J, Swanson KV: Invasion of human mucosal epithelial cells by Neisseria gonorrhoeae upregulates expression of intercellular adhesion molecule I (ICAM-I). Infect Immun 1999, 67:1149-1156.

25. Moulder JW: Comparative biology of intracellular parasitism. Microbiol Rev 1985, 49:298-337.

26. Tang P, Rosenshine I, Finlay BB: Listeria monocytogenes, an invasive bacterium, stimulates MAP kinase upon attachment to epithelial cells. Mol Biol Cell 1994, 5:455-464.

27. Vaneechoutte M, Dijkshoorn L, Tjernberg I, Elaichouni A, de Vos P, Claeys G, Verschraegen G: Identification of Acinetobacter genomic species by amplified ribosomal DNA restriction analysis. J Clin Microbiol 1995, 33: I I-I5.

28. Lee JS, Lee JC, Lee CM, Jung ID, Jeong YI, Seong EY, Chung HY, Park YM: Outer membrane protein A of Acinetobacter baumannii induces differentiation of $\mathrm{CD4}^{+} \mathrm{T}$ cells towards a Th I polarization phenotype through the activation of dendritic cells. Biochem Pharmacol 2007, 74:86-97.

29. Hanly WC, Artwohl JE, Bennett BT: Review of polyclonal antibody production procedures in mammals and poultry. ILARJ 1995, 37:93-118.
Publish with BioMed Central and every scientist can read your work free of charge

"BioMed Central will be the most significant development for disseminating the results of biomedical research in our lifetime. "

Sir Paul Nurse, Cancer Research UK

Your research papers will be:

- available free of charge to the entire biomedical community

- peer reviewed and published immediately upon acceptance

- cited in PubMed and archived on PubMed Central

- yours - you keep the copyright

Submit your manuscript here:

http://www.biomedcentral.com/info/publishing_adv.asp
BioMedcentral 